\title{
Relación médico-industria y los conflictos de interés: aspectos históricos y normativos, impactos negativos y propuestas
}

\author{
Giuseppe Genta-Mesa', Iván D. Flórez²,3
}

\section{RESUMEN}

El presente artículo revisa de manera narrativa y general la relación entre los médicos y la industria (RMI); se incluye a la industria farmacéutica, de dispositivos, de suplementos y los conflictos de intereses que surgen de dicha relación. Se aborda el tema mediante algunas definiciones y un breve recuento histórico de cómo la RMI se ha convertido en un problema importante en el ejercicio médico y los sistemas de salud.

El artículo expone los diversos roles dentro de los escenarios más comunes en los que ocurre la RMI: en la investigación biomédica, las guías de práctica clínica, la educación médica continua, la visita médica, los líderes de opinión, así como en la interacción directa, la exposición indirecta y la que denominamos exposición temprana. Se mencionan, posteriormente, algunas técnicas que utiliza la industria para modificar los hábitos de prescripción basadas en elementos de psicología social, así como el papel que ejercen los obsequios y otras estrategias de mercadeo.

Se revisó la literatura que muestra el impacto y el efecto que estas técnicas tienen en el ejercicio médico. Finalmente, se plantean algunas propuestas que se han desarrollado desde la normatividad y recomendaciones para afrontar el problema que podrían contribuir a mitigar los efectos negativos de una RMI muy estrecha. Estas propuestas son prohibir el contacto de los

\footnotetext{
Médico Pediatra, Profesor Asociado - Sección de Pediatría Social. Facultad de Medicina, Universidad de Antioquia, Medellín, Colombia.

Profesor Departamento de Pediatría y Puericultura, Universidad de Antioquia, Medellín, Colombia.

Department of Health Research Methods Evidence and Impact, McMaster University, Hamilton, Canada.
}

Correspondencia: Iván D. Florez; ivan.florez@udea.edu.co

Recibido: 7 de julio de 2018

Aceptado: 29 de octubre de 2018

Cómo citar: Genta-Mesa G, Flórez ID. Relación médico-industria y los conflictos de interés: Aspectos históricos y normativos, impactos negativos y propuestas. latreia. 2019 0ctDic;32(4):298-310. D0I. 10.17533/udea.iatreia.16. 
estudiantes con la industria, la inclusión del tema de conflictos de intereses (CI) en los currículos de medicina, financiación alternativa de la educación continua, declaración de conflictos como norma, campañas de concientización, uso de evidencia sintetizada y analizada transparente e independientemente, entre otras.

\section{PALABRAS CLAVE}

Conflictos de Intereses; Educación Médica; Ética Médica; Industria Farmacéutica

\section{SUMMARY}

Physician-industry relationship and conflicts of interest: Historical, normative aspects, negative impact and proposals

This article presents a narrative review of the relationship between physicians and industry (RPI), which includes pharmaceutical, devices and supplements' industries and the conflicts of interest that arise from this relationship. It introduces the problem through some definitions and a brief history review of how RPI has become a major problem in medical practice and health systems.

The article discusses the different roles within the most common scenarios in which RPI occurs: biomedical research, clinical practice guidelines, continuing medical education, medical visits, opinion leaders, as well as in direct interaction, indirect exposure, and what we call, an early exposure. Some techniques used by industry to modify prescription habits based on elements of social psychology, are described, as well as the role of gifts and other marketing techniques on prescriptions.

We review literature that shows the impact and effect these techniques have on medical practice. Finally, some initiatives, codes and recommendations that have been developed to address the problem and which could contribute to mitigate the negative effects of a very close RPI. The possible actions are: banning any contact between industry and students, inclusion of conflict of interests into the medical curricula, alternative financial support to continuous education, making the disclosure of conflicts of interests mandatory, launching awareness campaings among physicians, and the use of synthesized evidence that have been transparently and independently assessed, among others.

\section{KEY WORDS}

Conflict of Interest; Drug Industry; MedicaI, Education; Medical, Ethics

\section{INTRODUCCIÓN}

La relación entre los médicos y la industria (RMI) ha generado siempre un permanente debate ${ }^{(1)}$. La preocupación deriva de lo inconveniente que resulta el contacto estrecho que lleva a conflictos de interés (CI), que pueden afectar el comportamiento médico y la toma de decisiones en salud ${ }^{(2-9)}$. Los CI aparecen cuando dos o más intereses se contraponen: los beneficios del paciente (o del sistema sanitario) y los intereses de la industria ${ }^{(10)}$.

Se considera que existen CI cuando "un interés de tipo secundario, motivado por un grupo de condiciones, puede influir de manera indebida en su juicio profesional con relación a su interés primordial o primario que en su caso es el bienestar y cuidado del paciente" ${ }^{(11)}$.

Los CI han persistido en el tiempo, ganando relevancia en las últimas décadas. Recientemente, la revista JAMA dedicó un número completo a tratarlos desde diferentes aspectos ${ }^{(12)}$, evaluando su impacto en distintas áreas sanitarias ${ }^{(13-18)}$. Lamentablemente, en la literatura médica en lengua castellana son escasas las revisiones que tratan el tema con profundidad. El objetivo de esta revisión narrativa es presentar un recuento histórico, describir las principales características de la RMI, detallar la evidencia que existe sobre su impacto en tomar decisiones y mencionar algunas estrategias normativas desarrolladas para mitigar sus efectos.

Denominamos relación médico-industria (RMI) a toda la interacción directa o indirecta, entre el personal médico y la industria. Al referirnos a la industria, 
incluimos la farmacéutica, pero igualmente a las industrias de alimentos, suplementos nutricionales, dispositivos y biotecnología.

\section{BREVE HISTORIA}

Desde hace más de un siglo se conoce lo inapropiado de que la industria sea la fuente del conocimiento médico, al usar fuentes comerciales y promocionando productos en revistas médicas ${ }^{(19)}$. En 1908, Simmons (citado en Rodwin, MA), presidente de American Medical Association (AMA), afirmó “... el hecho de que la empresa Abbot Alkaloidal Company invierta miles de dólares en la publicidad de sus productos en diversas revistas médicas con artículos originales y testimonios, podría explicar por qué fueron publicadas" ${ }^{199}$. En 1929, (citado en Rodwin, MA), conferencistas de un simposio de la AMA expresaron la necesidad de reemplazar al representante de ventas (RV) como el principal informador sobre los medicamentos por fuentes no comerciales, $y$ criticaron los vínculos financieros entre la industria, los médicos y las revistas médicas que permitían la publicidad en sus páginas ${ }^{(19)}$.

May planteó en 1961 que la RMI deriva en una promoción de medicamentos disfrazada de educación continua. Afirmó que alogunos anuncios publicitarios de revistas tenían referencias sin contexto, falsas, engañosas o sin fundamento y propuso separar las funciones de los médicos y la industria ${ }^{(20)}$.

El tema siguió mencionándose por décadas, pero no fue sino hasta la década pasada en que se retomó el interés en la literatura. Wazana hizo una revisión no sistemática de los estudios, evaluó la RMI describiendo cómo la interacción de médicos con RV era bastante aceptada por los facultativos, asociándose con cambios en las prescripciones y en el incremento de solicitudes para incluir medicamentos en formularios hospitalarios. También, reseña cómo la asistencia de profesionales a reuniones de educación médica continua (EMC) con expositores escogidos y pagados por la industria u organizadas por sociedades científicas, pero con inscripciones, viajes y hospedaje financiados por la industria, se asociaban con una mayor prescripción de medicamentos del patrocinador ${ }^{(21)}$. Otros estudios han corroborado dichos resultados, destacando el efecto sobre la prescripción de medicamentos de marca, a pesar de disponer de genéricos igualmente eficaces ${ }^{(22-26)}$.

\section{ACTUACIONES DE LA INDUSTRIA FARMACÉUTICA EN DIFERENTES ESCENARIOS}

Detallaremos ocho escenarios que resumirán las situaciones más frecuentes en la práctica y en la literatura médica con sus potenciales efectos.

1. Investigación biomédica: la industria participa activamente en una o varias de las fases de un proyecto de investigación financiado por ellos: concepción y diseño del estudio (por ejemplo, selección del comparador, selección de desenlaces no importantes para los pacientes), recolección y análisis de datos y la publicación final de sus resultados en artículos científicos. Precisamente, por tener el control sobre estas fases, tiene también el poder decisorio sobre la eventual publicación de los resultados en artículos científicos.

Se ha descrito como en muchos casos, la industria se abstiene de publicar los resultados de estudios de sus propios medicamentos porque estos resultaron desfavorables, a lo que se le denomina sesgo de publicación ${ }^{(27-30)}$.

Por ejemplo, Jones y col. ${ }^{(27)}$ analizaron cómo 4 años después de haber sido terminados 585 estudios registrados en la plataforma de ensayos clínicos (EC) (www. clinicaltrials.gov), el 29 \% de ellos estaban sin publicar. Los EC financiados por la industria son más propensos a no publicarse que aquellos sin su financiación, y una tercera parte de ellos nunca serán publicados. Los EC con resultados positivos (con diferencias estadísticamente significativas, o si el medicamento resultó mejor que la comparación) tienen una mayor posibilidad de ser publicados que aquellos con resultados negativos ${ }^{(28)}$.

En una revisión sistemática, Bekelman y col. ${ }^{(29)}$ encontraron que los estudios relacionados con la industria tenían casi 4 veces más opciones de concluir favorablemente hacia su medicamento en comparación con aquellos no financiados por esta. Se encontró que aproximadamente una cuarta parte de los autores tenían nexos con la industria y dos tercios de 
los centros académicos poseían acciones en la industria. Krimsky y col. ${ }^{(30)}$ reportaron que un tercio de los artículos publicados en Massachussets tenían intereses financieros con sus patrocinadores. Por su parte, Bhandari y col. ${ }^{(31)}$ encontraron mayor posibilidad de obtener hallazgos significativos, al comparar estudios financiados con estudios no financiados por la industria $(\mathrm{OR}=2,3 ; \mathrm{IC} 95 \% 1,3 ; 4,1)$.

Goldacre ${ }^{(32)}$ resalta cómo la industria interfiere en la publicación de resultados; si estos son desfavorables, suministra datos parciales ocultando los resultados negativos. Los médicos “des-informados”, asumen conductas inadecuadas, arriesgándose al prescribir medicamentos potencialmente dañinos ${ }^{(1,32,33-34)}$. En la Tabla 1 se resumen los posibles efectos de la industria en la investigación en publicaciones científicas, guías de práctica clínica y educación médica continua, tales como los sesgos de publicación, sobreestimación del efecto del medicamento, comparación con placebos, evaluación de desenlaces no importantes, financiación de las guías de práctica clínica, la participación indirecta, la financiación indirecta, la definición de contenidos y temáticas, la instalación de estands, el lanzamiento de nuevos medicamentos, el pago a expositores y la diseminación de información, entre $\operatorname{otros}^{(22,28,33)}$.

\section{Tabla 1. Posibles efectos de la industria farmacéutica en la investigación, las publicaciones, las guías de práctica clínica y la educación médica continuada}

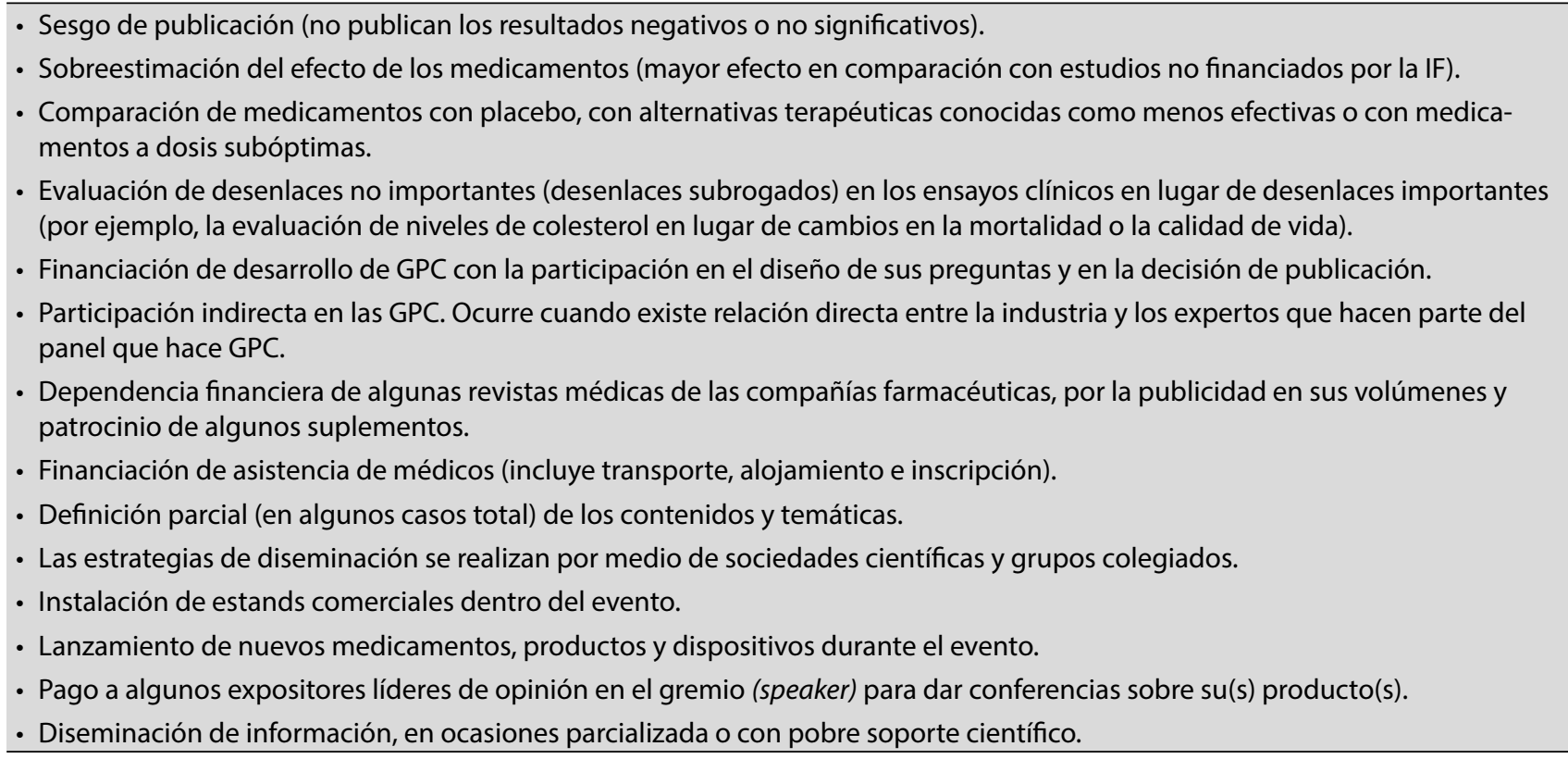

IF: Industria farmacéutica; GPC: Guías de práctica clínica; EMC: Educación médica continua. Fuente: elaboración propia referencias (1,2,4,22,28)

2. Guías de práctica clínica: (GPC) Cuando son desarrolladas, transparente y sistemáticamente, proveen recomendaciones basadas en evidencia, apoyan las decisiones sanitarias y evitan prescripciones inadecuadas o innecesarias ${ }^{(35)}$. Desafortunadamente, las GPC son afectadas por los CI. Neuman y col. ${ }^{(35)}$ encontraron que el $48 \%$ de expertos en 14 GPC para diabetes y dislipidemia declararon CI, y en el $11 \%$ de quienes no declaraban CI, se encontró alguno. Además, GPC con financiación gubernamental eran menos propensas a tener CI que las financiadas por otras fuentes.

Muchas organizaciones desarrollan GPC con estricto control de los CI en su participación para garantizar la credibilidad ${ }^{(35)}$, pero esto no ocurre en todos los casos. Es importante recomendar a los usuarion de las GPC verificar que estas cuenten con una declaración 
de CI, y que esta cumpla con independencia editorial del ente financiador antes de usarlas ${ }^{(36)}$. Las GPC que tengan $\mathrm{CI}$ importantes o son dependientes de financiación de la industria, no deben ser consideradas para su uso en la práctica clínica.

3. Educación médica continua (EMC): la EMC difunde la investigación biomédica y contribuye a la formación continua de los profesionales de la salud. Sin embargo, este objetivo fundamental se afecta por la RMI. La industria financia la mayoría de los eventos académicos (desde pequeños simposios hasta prestigiosos congresos mundiales), organiza, prioriza y decide los contenidos de estos eventos. ${ }^{(1,2,4,22,28)}$. En la (Tabla 1), se describen algunas acciones identificadas en este escenario.

4. Visita médica (VM): es la vía de acceso directa al médico, con el objetivo de diseminar información, obsequios e invitaciones a eventos. Suele ser breve, un $\mathrm{RV}$ acude al consultorio $\mathrm{u}$ hospital repetidas veces, informando al médico sobre sus productos, con evidencia científica seleccionada (sin evaluación de calidad) que le favorece con información, no siempre exacta, completa y equilibrada, sin mencionar los efectos perjudiciales y el costo comparativo del mismo, dando obsequios que generarán en el médico un deseo inconsciente de retribuir dichos favores. Las VM son muy frecuentes (son una práctica universal), percibidas como una relación normal e incluso necesaria que forjará relaciones mutuamente beneficiosas en el futuro ${ }^{(21,32)}$. Los RV utilizan diversas técnicas durante la $\nabla M$ que buscan persuadir al profesional a prescribir sus medicamentos ${ }^{(37)}$. En la (Tabla 2) se listan algunas actuaciones de la industria durante la VM.

\section{Tabla 2. Actuaciones de la industria farmacéutica en la visita médica y los obsequios inapropiados de la industria farmacéutica hacia el personal médico}

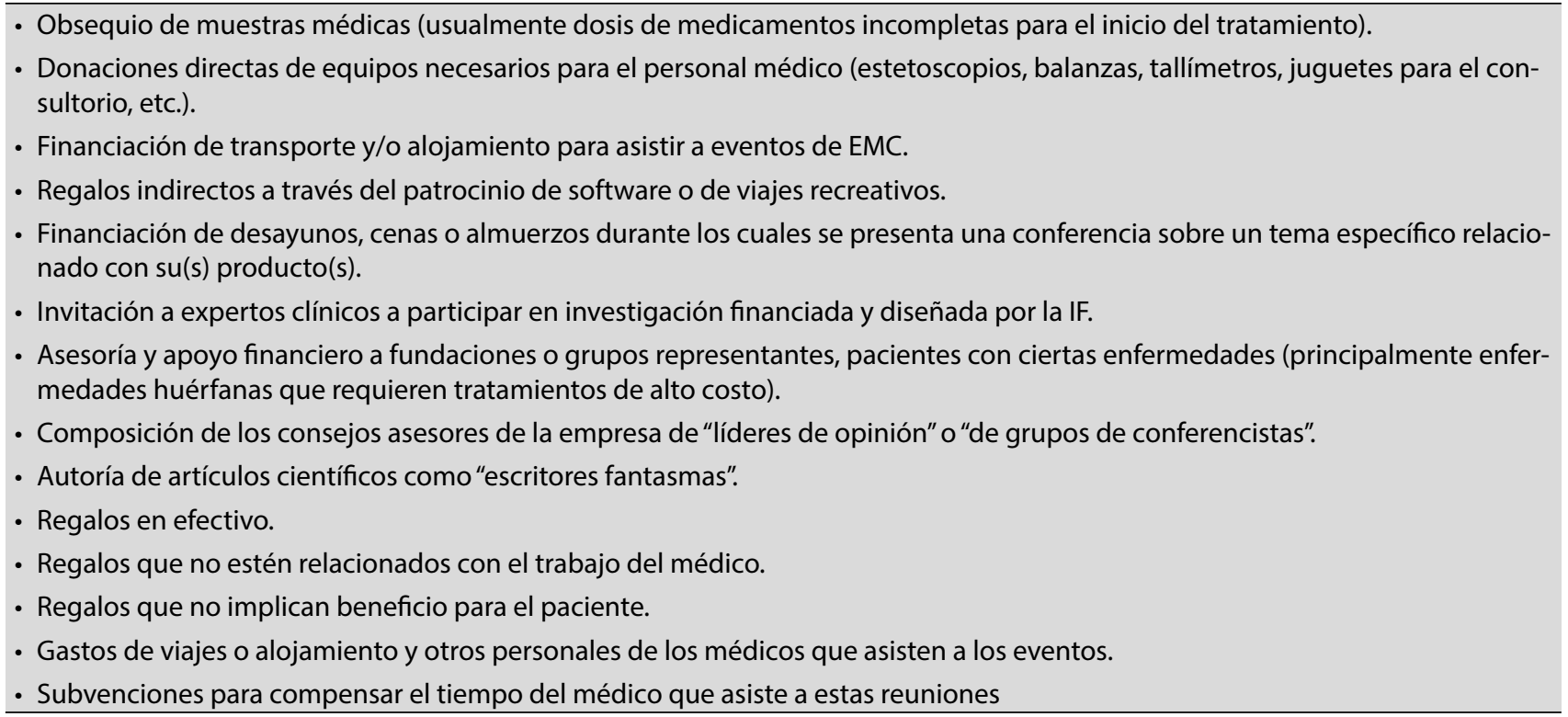

EMC: Educación médica continua; IF: Industria farmacéutica; Fuente: elaboración propia, referencias $(1,9,32,33,37)$

5. Líderes de opinión: una estrategia común de la IF es trabajar con los líderes de opinión (LO). Expertos reconocidos en una especialidad, financiados por la IF para influir en los médicos con charlas o asuntos específicos, la industria invita a grupos de médicos a escucharlas por parte de LO. Promueven sus productos directa o indirectamente; la industria asume los gastos de alquiler del salón/restaurante, comida $y$ honorarios del conferencista. Una tarea fundamental del RV es convertir a LO en conferencistas por 
procesos graduales con pequeños regalos que establecen la confianza y consolidan paulatinamente la relación, se vuelven conferenciantes speakers ${ }^{(38)}$. A veces LO prestan su nombre como autores de artículos previamente escritos por la industria, recibiendo honorarios. Se denominan escritores fantasmas, generan conductas éticamente inapropiadas para una publicación científica ${ }^{(39)}$.

6. Interacción financiera directa: es la relación económica inmediata entre médicos e industria. Ocurre cuando el médico Iabora como empleado, posee acciones o participación de capital, es consultor (ocasional o permanente), hace parte de consejos asesores de la empresa, es conferenciante (speaker) o aparece como un escritor fantasma para $\operatorname{la} \operatorname{IF}^{(1,32,33,37}$ 39) Estas interacciones generan CI financieros directos y un sentido de lealtad a las compañías empleadoras $(1,32,37,40)$. En estos casos, el profesional tiene una dependencia económica en la cual claramente se beneficia si la empresa tiene un lucro, y por lo tanto, la objetividad en las decisiones o comentarios al respecto de productos de dicha empresa se pierde. Estos CI tendrán importancia cuando el médico que tiene relación financiera directa toma decisiones sanitarias con pacientes o desarrolla artículos científicos o GPC.

7. Exposición indirecta del médico con la industria: los roles de la industria en otras actividades indirectas incluyen la financiación de asociaciones de pacientes (que influyen en médicos y son quienes deciden cuáles medicamentos prescribir o financiar), el apoyo financiero de sociedades científicas y campañas de desprestigio hacia medicamentos genéricos ${ }^{(1,9,37)}$. Aunque son menos estudiadas y más difícil de investigar, también tienen el potencial de afectar los hábitos de formulación.

8. Exposición temprana: La RMI existe desde las escuelas de medicina; una revisión sistemática encontró que los estudiantes de universidades que restringían a los RV eran más escépticos y críticos con la información que aquellos estudiantes sin restricciones ${ }^{(17)}$. Larkin reportó una reducción modesta pero significativa en prescripción de medicamentos de marca cuando restringían el contacto de los estudiantes con la IF (41). La exposición temprana puede ser un factor que explique la percepción de la RMI como algo natural. Cuando terminan sus estudios prescriben más medicamentos de las empresas que los visitaron y a cuyos eventos asistieron patrocinados ${ }^{(32,38)}$.

\section{CUÁL ES EL EFECTO DE ESTA INTERACCIÓN}

Hasta acá hemos descrito las características de la interacción industria-médicos, directa o indirectamente. Es lógico pensar que la existencia de interacción no implica, necesariamente, un efecto negativo sobre los pacientes o sobre el sistema. Cabe mencionar que, los médicos en general, consideran que las anteriores técnicas tienen poca o nula influencia sobre ellos ${ }^{(2-4,37,38)}$.

En dos universidades de Estados Unidos (EE. Uu.), el $85 \%$ de los estudiantes de medicina consideraron inapropiado que un funcionario público aceptara regalos de USD $\$ 50$ de la industria, pero solo el $46 \%$ consideró inadecuado que ellos mismos lo aceptaran ${ }^{(42)}$. Además, el $61 \%$ de los médicos residentes de medicina interna de un hospital en EE. UU. consideraban que las VM y los regalos no influían en su propia prescripción y, el 84 $\%$ consideró que esta sí influía en sus colegas ${ }^{(43)}$. Existe evidencia que muestra que a mayor interacción con la $\nabla M$, esta se percibe con menos efectos sobre el receptor de Ia misma; es decir, a mayor intensidad de la VM los médicos con mayor frecuencia la perciben como algo normal e inocuo ${ }^{(23,25,26)}$.

Hodges describió que, a mayor número de obsequios y artículos promocionales recibidos por los residentes, más probabilidad tenían de considerar que la RMI no afectaba sus prescripciones. Los residentes de los primeros dos años tenían tres veces más contacto con $\mathrm{RV}$, consideraban con mayor frecuencia que las visitas revestían un carácter educativo comparado con residentes mayores ${ }^{(44)}$. Adicionalmente, los médicos consideran que pequeños obsequios pueden ser éticamente más aceptables que los más costosos y no afectan su juicio con relación a un prođucto ${ }^{(23,26,45,46)}$.

Existe evidencia que los regalos, aunque pequeños pueden impactar los hábitos de prescripción. Se ha planteado que incluso cuando los individuos tratan de ser objetivos, sus juicios están sujetos a un sesgo inconsciente no intencional de autocomplacencia (conveniencia) ${ }^{\left({ }^{45}\right)}$. En el contexto de una $\nabla M$, se combina el efecto de la forma en que se transmite el mensaje con el efecto del obsequio ${ }^{(45)}$; por ende, la suposición de que la interacción estrecha y las dádivas no afectan la conducta del médico, es debatible (46). Se ha encontrado una asociación entre VM, obsequios y el aumento en la formulación de medicamentos registrados ${ }^{(21,23-26,45-46)}$. Waud ${ }^{(47)}$, coincidiendo con 
otros autores ${ }^{(23,26,44,46)}$, plantea que las $\nabla M$ repetidas inciden en la prescripción y describe cómo se presentan nuevos CI derivados de las conductas de algunos médicos, como compartir honorarios o recibir comisiones por formulaciones, derivar pacientes a colegas o autoderivar pacientes o exámenes de laboratorio a instituciones donde son accionistas ${ }^{(47)}$.

Todas estas influencias tienen un impacto que puede resultar perjudicial para los pacientes y la sociedad. Sobre los pacientes porque existirán muchas ocasiones en los que las decisiones médicas serán influenciadas por la selección de un medicamento que podría no ser el más efectivo o, resultar incluso, peligroso, dado que esta relación tan estrecha trae consigo decisiones que pueden apartarse de la evidencia o del tratamiento aceptado ${ }^{(48)}$.

Adicionalmente, existe un impacto indirecto sobre la sociedad; las decisiones clínico-terapéuticas bajo esta relación pueden conducir al uso indiscriminado de tratamientos inefectivos o efectivos, pero menos económicos, afectando así en forma directa el uso racional de los recursos de un sistema de salud. Esto implicará un desvío de los recursos que se espera sean invertidos en decisiones adecuadas, transparentes y más beneficiosas para otros pacientes ${ }^{(48)}$.

\section{CÓMO SE EXPLICA LA INFLUENCIA}

Algunas aproximaciones en la búsqueda de explicación para esta situación se basan en la ilusión de que la industria es generosa $y$ desinteresada con los médicos ${ }^{(49)}$. Cialdini ${ }^{(50)}$ ha estudiado seis principios de influencia y su impacto en el mercadeo desde la psicología social: reciprocidad, compromiso, prueba social, simpatía, autoridad y escasez, (tabla 3). Estas técnicas son aplicadas por la industria por medio de sus RV, pero usualmente los médicos no reconocen su vulnerabilidad a las influencias, muy probablemente debido a los sesgos por conveniencia y a sus racionalizaciones ${ }^{(49,50-52)}$.

\section{Tabla 3. Principios de influencia}

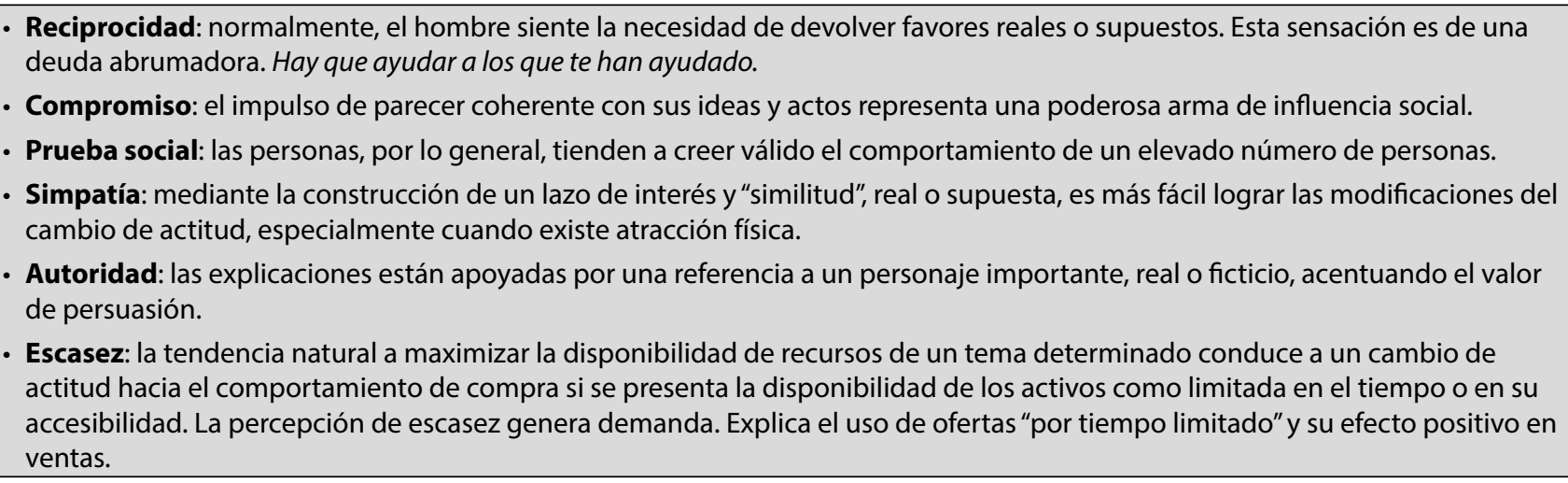

Fuente: elaboración propia; referencias ${ }^{(49,59)}$.

Se ha descrito que en general, las personas son más fuertemente influenciadas por mensajes entregados con confianza, y no se toman la molestia de comprobar la veracidad de estos mensajes si ello requiere esfuerzo o dinero, además, suelen aferrarse a la primera información que reciben, incluso después de descubrir que esta es inexacta o no pertinente ${ }^{(32,33,49,52)}$. Cialdini propone educar a profesores $y$ estudiantes sobre la psicología social subyacente al mercadeo y cómo hacerle resistencia, plantea desarrollar políticas organizacionales enéroicas en las instituciones académicas para independendizarlas de la industria ${ }^{(50,53,54)}$.

La psicología social postula que las personas tienen un punto ciego al propio sesgo, es decir, hay más propensión a identificar la existencia de sesgos cognitivos y motivacionales en los demás que en sí mismos ${ }^{(55)}$. 
Esta situación no es exclusiva del campo médico, esto ocurre en todas las áreas del conocimiento. La situación particular en medicina, es que comúnmente se cree que poseen objetividad científica, sin embargo, su tendencia a aceptar obsequios genera una disonancia cognitiva, es decir, un escenario de incomodidad o angustia que surge de la discrepancia entre creencias contradictorias, o entre creencias y comportamientos ${ }^{(56)}$. Las personas manejan o reducen esta disonancia cambiando una de las creencias, opiniones o comportamientos disonantes, reduciendo la importancia de uno de los factores discordantes (mediante negación), o agregando elementos consonantes que resuelvan o reduzcan la disonancia ${ }^{(49,51,56)}$.

Chimonas y col. identificaron varias negaciones y racionalizaciones para reducir la disonancia cognitiva en Ias RMI. Las negaciones incluyen evitar pensar en el CI, rechazar la idea de que la RMI afecta su comportamiento $y$, desconocer o universalizar la responsabilidad por los problemas que surgen de los $\mathrm{CI}$ (por ejemplo: “...siempre hay un conflicto de intereses...") ${ }^{(57)}$. Las racionalizaciones aseguran la existencia de técnicas que ayudan a mantener la imparcialidad $y$ estimar que las reuniones con RV son educativas y benefician a los pacientes ${ }^{(49,57)}$.

Es común que los médicos desconozcan el impacto de los sesgos inconscientes al tomar decisiones terapéuticas, por eso racionalizan y creen solo lo que quieren creer ${ }^{(51)}$. El prejuicio en el sentido de un juicio preformado puede surgir de muchas fuentes distintas al interés financiero. ${ }^{(16)}$ Las amistades, Ias asociaciones institucionales, el estudio previo y la reflexión, el conocimiento de un campo y la experiencia de vida, son factores que pueden predisponer a las personas a aceptar, rechazar o interpretar la evidencia de manera particular ${ }^{(16)}$. Las creencias de los médicos sobre los fármacos están asociadas más fuertemente con el material presentado por un RV que por los materiales científicos, así los médicos le asignen mayor peso a estos últimos ${ }^{(52)}$.

\section{REGULACIONES E INICIATIVAS}

El abordaje ideal para el problema sería que el personal médico lograra un autocontrol y redujera la interacción con la IF. Dada la evolución de esta preocupante situación, es necesario generar regulaciones gubernamentales y directrices éticas. A principio de Ios noventa el Servicio de Salud y Servicios Humanos de EE. UU., publicó dos documentos importantes ${ }^{(58 .}$ ${ }^{59)}$. En el primero, analizaron 109 anuncios de propaganda médica, encontraron que el $60 \%$ de ellos eran "pobres" o "inaceptables" en términos de calidad y presencia de referencias científicas, que la mitad tenía poco o ningún valor educativo, y que el $59 \%$ no conducía a una prescripción apropiada en médicos desinformados ${ }^{(58)}$. El segundo documento ${ }^{(59)}$, especifica que el $82 \%$ de los médicos recibieron obsequios (Iapiceros, libretas, muestras médicas) o pagos, en promedio unas 28 ocasiones por médico al año, y que la industria otorgaba más obsequios con más formulaciones. La asociación de investigadores y productores farmacéuticos en EE. UU. emitió un código de interacciones con profesionales sanitarios, especificando que los materiales promocionales deben ser precisos y no engañosos, hacer afirmaciones solo cuando esté adecuadamente justificado, reflejar riesgos $y$ beneficios y, ser compatibles con los requerimientos de la Agencia de administración de alimentos y drogas (del inglés Food and Drug Administration, FDA) ${ }^{(60)}$.

Estas normativas no son globales y no implican necesariamente un cambio. Otras propuestas han tratado de promover la transparencia mediante la declaración obligatoria de las interacciones financieras entre médicos e IF. En EE. UU., el llamado Sunshine Act-19 obligó a reportar los ingresos económicos y los obsequios recibidos por médicos, sociedades, investigadores, gestores, organizaciones de pacientes y ligas de consumidores por parte de la industria. Dicha información está disponible en un sitio web público desde $2014^{(61)}$. Es un paso importante, insuficiente aún, hacia la restitución de la confianza en los médicos. Luego de casi 4 años de implementarse se observa una pequeña disminución en las interacciones financieras entre los médicos y la industria ${ }^{(25,62)}$. Regulaciones similares son obligatorias en Francia ${ }^{(63)}$ y voluntarias en el Reino Unido ${ }^{(64)} y$ España. En esta última, fueron formuladas dichas recomendaciones en 2005 por el Consejo General de Colegios Médicos de España ${ }^{(65)}$, y complementadas en el 2017 con la Primera Guía de Buenas Prácticas de Información Médica en la Industria Farmacéutica ${ }^{(66)}$. En México, por su parte, en el 2016 se realizaron recomendaciones similares con el Comité de Ética y Transparencia en la Relación Médico-Industria ${ }^{(67)}$. 
En Colombia, el Ministerio de Salud y Protección Social, expiđió la resolución 2881 de 2018, la cual creó el Registro de Transferencias de Valor entre Actores del Sector Salud y la Industria farmacéutica y de tecnologías en salud, denominado RTVSS ${ }^{(68)}$. EI RTVSS obliga a la industria a reportar toda transferencia de valor que se ejecute desde la industria a los profesionales de la salud, cuando esta supere el valor de un salario mínimo mensual vigente (SMMV) en Colombia por semestre (al momento de escribir este artículo el SMMV equivale a 781.242 pesos colombianos, o 272.75 dólares estadounidenses). Esta resolución pretende hacer transparentes las relaciones entre la IF y los profesionales sanitarios. No se conocen otras iniciativas de leyes o códigos en Latinoamérica.

Han surgido en el mundo organizaciones preocupadas por la estrecha RMI como Unbranded doctor, No Free Lunch, Grazie pago io, entre otras. En Chile apareció Médicos Sin Marca, cuya rama en Colombia inició recientemente (https://medicossinmarca.co/). Estas iniciativas, sin poder regulatorio ni decisorio directo, ayudan a diseminar la información relacionada con la RMI y crean conciencia y trasparencia en los profesionales sanitarios.

\section{POSIBLES SALIDAS}

Un primer paso, debería ser la transparencia: que los profesionales e instituciones de salud reporten toda relación con la IF. Para médicos e instituciones, la regla general debería ser: si hacer públicos ingresos u obsequios les supone un problema, no deberían aceptarlos. Una segunda regla es recordar que viajes, comidas y conferencias, al final, los acaban pagando los pacientes, el sistema de salud y los ciudadanos con

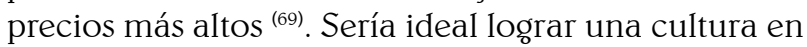
la que recibir obsequios generase alguna veroüenza y no gratitud, esto reduciría los $C{ }^{(69,70)}$. Steinbrook propone que los médicos, en lugar de aceptar invitaciones deberían presionar a la IF para invertir más dinero en auténtica investigación sobre seguridad, eficacia y asequibilidad a los medicamentos ${ }^{(69)}$.

Brennan y col., proponen que las instituciones ejerzan regulaciones más fuertes, prohibiendo actividades que constituyen CI serios y desarrollen investigaciones independientes de la IF ${ }^{(53)}$. Wayne y col. sugieren no aislar a los estudiantes de las interacciones con la industria, se debe buscar inculcar sensibilidad profesional para trabajar eficazmente contra intereses externos ${ }^{(17)}$.

Abordajes más extremos han sido planteados. Angell propone una completa supresión de la industria como fuente de EMC y políticas de restricción al mercadeo farmacéutico en hospitales universitarios ${ }^{(6,54)}$.

En nuestro medio, Gómez Córdoba y col. (2007) han abordado los dilemas en las relaciones entre la industria farmacéutica y los profesionales de la salud, las tensiones generadas en esta relación y la consideran como posible y necesaria, proponiendo la autorregulación como la alternativa de acción más constructiva ${ }^{(71)}$. Lo y Grady recomiendan que todas las universidades prohíban la participación de los profesores como conferencistas para la IF ${ }^{(26)}$. La RMI es heterogénea y compleja, por lo que se requieren diversas estrategias a corto y largo plazo. Se han utilizado muchas, pero su provecho puede ser limitado, aunque útil para disminuir los efectos nocivos. Moynihan ${ }^{(4)}$ propone implementar una serie de acciones, que han demostrado resultados (Tabla 4). ${ }^{(4,15,46,47,54,69,70)}$.

\section{CONCLUSIONES}

Entender los CI que ocurren por una estrecha RMI es fundamental para que estos sean visibilizados, ellos afectan la autonomía del médico, los pacientes y las finanzas de los servicios de salud. La RMI puede ser directa ( $\nabla M$, junto con obsequios), pero también puede ser indirecta (acceso a GPC, estudios clínicos con CI e influencia sobre las asociaciones de pacientes, entre otras).

Es recomendable desarrollar propuestas diversas con el objetivo de disminuir los efectos nocivos. Algunas propuestas normativas (bases de datos gubernamentales de reporte de transferencias de valor) han sido implementadas en algunos países (incluida, muy recientemente, Colombia). Propuestas más específicas que requieren de esfuerzos institucionales y académicos son la prohibición del contacto de los estudiantes de medicina con la IF, la inclusión obligatoria del tema de CI en los currículos de medicina de las universidades, la búsqueda de fuentes de financiación alternativas para la educación médica continua y el desarrollo de guías de práctica clínica. La declaración 


\section{Tabla 4. Propuestas de acción para afrontar la relación médicos-industria farmacéutica}

- Lograr que la educación y fuentes de información científica sean independientes de la IF.

- Universidades y hospitales deberían poner fin a los desayunos, almuerzos y cenas patrocinados por la IF.

- Prohibir la existencia de representantes de ventas de medicamentos en hospitales universitarios.

- Las restricciones o prohibiciones de eventos educativos sean financiados por la IF.

- Prohibir a individuos u organizaciones que tengan conflictos de intereses serios para que no acrediten educación médica continua.

- Generar campañas para eliminar la aceptación de todos los regalos y viajes.

- Generar campañas para poner fin a la aceptación de los honorarios de los expositores en las conferencias educativas.

- Reducir y, eventualmente, eliminar, la dependencia de las asociaciones médicas de la financiación de la IF.

- Las revistas médicas deben reducir, hasta eliminar la financiación proveniente de la IF.

- Las llamadas a establecer "fideicomisos ciegos" a nivel institucional para manejar de forma independiente a los fondos externos.

- Llamar a nuevas entidades nacionales para llevar a cabo las investigaciones que tengan interés para el público en general.

- Llamar a los miembros de los comités de reglamentación y de asesoramiento para que eviten conflictos de interés.

- Pagar a los médicos sobre una base salarial sin incentivos para el volumen de servicios rendido.

IF: Industria farmacéutica. Fuente: elaboración propia, referencias ${ }^{(4,15,46,47,53,70)}$

de CI como norma (en publicaciones científicas, presentaciones en congresos, atención de pacientes $y$ mediante bases de datos gubernamentales), campañas de concientización con los médicos y estudiantes, el uso de la evidencia sintetizada y analizada de forma transparente e independiente en la toma de decisiones, entre otras.

En síntesis, dada la enorme dificultad para escapar a la disonancia cognitiva (problema de todo ser humano), proponemos tomar prudente distancia en esta relación para que no sea tan estrecha, dado que es imposible no tener algún contacto, y así convivir con la IF.

\section{CONFLICTOS DE INTERESES}

Ninguno por declarar.

\section{REFERENCIAS BIBLIOGRÁFICAS}

1. Field MJ, Lo B, editors. Conflict of interest in medical research, education, and practice. Washington, EE. Uu: National Academies Press; 2009; p 414.

2. Orlowski JP, Wateska L. The effects of pharmaceutical firm enticements on physician prescribing patterns: there's no such thing as a free lunch. Chest. 1992;102(1):270-3.
3. Lexchin J. Interactions between physicians and the pharmaceutical industry: what does the literature say? CMAJ. 1993;5(10):1401-7.

4. Moynihan R. Who pays for the pizza? Redefining the relationships between doctors and drug companies. 2: Disentanglement. BMJ. 2003 May 31;(7400):1193-6. DOI 10.1136/bmj.326.7400.1193.

5. Fugh-Berman A, Ahari S. Following the script: how drug reps make friends and influence doctors. PLoS Medicine. 2007;4(4):e150. DOI 10.1371/journal. pmed.0040150.

6. Santa Cruz P. La industria farmacéutica y su influencia en la práctica clínica. Rev GPU. 2011;1:92-102.

7. DeAngelis CD. The influence of money on medical science. JAMA. 2006;296(8):996-8. DOI 10.1001/ jama.296.8.jed60051.

8. Campbell EG. Doctors and drug companies-scrutinizing influential relationships. N Engl J Med. 2007;357(18):1796-7. DOI 10.1056/NEJMp078141.

9. Abbasi K, Smith R. No more free lunches: patients will benefit from doctors and drug companies disentangling. BMJ. 2003;326(7400):1155-6. DOI 10.1136/ bmj.326.7400.1155.

10. Lama A. El médico y los conflictos de intereses. Rev Med Chil. 2003;131(12):1463-8. DOI 10.4067/S003498872003001200015 . 
11. Thompson DF. Understanding financial conflicts of interest. N Engl J Med. 1993;329(8):573-6. DOI 10.1056/ NEJM199308193290812.

12. Stead WW. The Complex and Multifaceted Aspects of Conflicts of Interest. JAMA. 2017;317(17):1765-7. DOI 10.1001/jama.2017.3435.

13. Fineberg HV. Conflict of interest: why does it matter? JAMA. 2017;317(17):1717-8. DOI 10.1001/jama.2017.1869.

14. Ornstein C. Public Disclosure of Payments to Physicians from Industry. JAMA. 2017;317(17):1749-50. DOI 10.1001/jama.2017.2613.

15. Larkin I, Loewenstein G. Business Model-Related Conflict of Interests in Medicine: Problems and Potential Solutions. JAMA. 2017;317(17):1745-6. DOI 10.1001/jama.2017.2275.

16. Flier JS. Conflict of Interest Among Medical School Faculty: Achieving a Coherent and Objective Approach. JAMA. 2017;317(17):1731-2. DOI 10.1001/ jama.2017.1751.

17. Wayne DB, Green M, Neilson EG. Teaching Medical Students About Conflicts of Interest. JAMA. 2017;317(17):1733-4. DOI 10.1001/jama.2017.2079.

18. Pizzo PA, Lawley TJ, Rubenstein AH. Role of Leaders in Fostering Meaningful Collaborations Between Academic Medical Centers and Industry While Also Managing Individual and Institutional Conflicts of Interest. JAMA. 2017;317(17):1729-0. DOI 10.1001/jama.2017.2573.

19. Rodwin MA. Drug advertising, continuing medical education, and physician prescribing: a historical review and reform proposal. J Law Med Ethics. 2016;38(4):80715. DOI 10.1111/j.1748-720X.2010.00534.x.

20. May CD. Selling drugs by "educating" physicians. J Med Educ. 1961;36(1):1-23.

21. Wazana A. Physicians and the pharmaceutical industry: is a gift ever just a gift? JAMA. 2000;283(3),373-80. DOI 10.1001/jama.283.3.373.

22. Neumann PJ, Sandberg EA, Bell CM, Stone PW, Chapman RH. Are pharmaceuticals cost effective? A review of the evidence. Health Aff (Millwood). 2000;19(2):92109. DOI 10.1377/hlthaff.19.2.92.

23. Grande D, Frosch DL, Perkins AW, Kahn BE. Effect of Exposure to Small Pharmaceutical Promotional Items on Treatment Preferences. Arch Intern Med. 2009;169(9):887-93. DOI 10.1001/archinternmed.2009.64.
24. DeJong C, Aguilar T, Tseng C-W, Lin GA, Boscardin WJ, Dudley RA. Pharmaceutical Industry-Sponsored Meals and Physician Prescribing Patterns for Medicare Beneficiaries. JAMA. 2016;176(8):1114-22. DOI 10.1001/jamainternmed.2016.2765.

25. Ornstein C. Public Disclosure of Payments to Physicians From Industry. JAMA. 2017;317(17):1749-50. DOI 10.1001/jama.2017.2613.

26. Lo B, Grady D. Payments to Physicians. JAMA. 2017;317(17):1719-20. DOI 10.1001/jama.2017.1872.

27. Jones CW, Handler L, Crowell KE, Keil LG, Weaver MA, Platts-Mills TF. Non-publication of large randomized clinical trials: cross sectional analysis. BMJ. 2013;347:f6104. DOI 10.1136/bmj.f6104.

28. Lexchin J, Bero LA, Djulbegovic B, Clark O. Pharmaceutical industry sponsorship and research outcome and quality: systematic review. BMJ. 2003;326(7400):116770. DOI 10.1136/bmj.326.7400.1167.

29. Bekelman JE, Li Y, Gross CP. Scope and Impact of Financial Conflicts of Interest in Biomedical Research. JAMA. 2003;289(4):454-65. DOI 10.1001/ jama.289.4.454.

30. Krimsky S, Rothenberg LS, Stott P, Kyle G. Scientific Journals and Their Authors' Financial Interests: A PiIot Study. Psychother Psychosom. 1998;67(4-5):194201. DOI 10.1159/000012281.

31. Bhandari M, Busse JW, Jackowski D, Montori VM, Schünemann H, Sprague S, et al. Association between industry funding and statistically significant pro-industry findings in medical and surgical randomized trials. CMAJ. 2004;170(4):477-80.

32. Goldacre B. Bad pharma: how drug companies mislead doctors and harm patients. London, Fourth Estate; 2012.

33. Blech J. Inventing disease and pushing pills: Pharmaceutical companies and the medicalisation of normal life. London: Routledge; 2006.

34 Ioannidis JPA. Are Medical Conferences Useful? And for Whom?. JAMA. 2012;307(12):1257-8. DOI 10.1001/ jama.2012.360.

35. Neuman J, Korenstein D, Ross JS, Keyhani S. Prevalence of financial conflicts of interest among panel members producing clinical practice guidelines in Canada and United States: cross sectional study. BMJ. 2011;343:d5621. DOI 10.1136/bmj.d5621. 
36. Florez Gomez ID, Montoya DC. Las guías de práctica clínica y el instrumento AGREE II. Rev Colomb Psiquiatr. 2011;40(3):563-76. DOI 10.1016/S00347450(14)60147-5.

37. Moynihan R. Who pays for the pizza? Redefining the relationship between doctors and drug companies. Entanglement. BMJ. 2003;326:1189-92. DOI 10.1136/ bmj.326.7400.1189.

38. Oldani MJ. Thick Prescriptions: Toward an Interpretation of Pharmaceutical Sales Practices. Med Anthropol Q. 2004;18(3):325-56. DOI 10.1525/ maq.2004.18.3.325.

39. Relman AS. Separating Continuing Medical Education From Pharmaceutical Marketing. JAMA. 2001;285(15):2009-12. DOI 10.1001/jama.285.15.2009.

40. National Institute Health and Care Excellence N. Policy on declaring and managing interests for NICE advisory committees [internet]. London: NICE; 2018 [accessed on March $8^{\text {th }}$, 2019]: Available from: https:// bit.ly/2SPnYxf

41. Larkin I, Ang D, Steinhart J, Chao M, Patterson M, Sah S, et al. Association Between Academic Medical Center Pharmaceutical Detailing Policies and Physician Prescribing. JAMA. 2017;317(17):1785-95. DOI 10.1001/jama.2017.4039.

42. Palmisano P, Edelstein J. Teaching drug promotion abuses to health profession students. J Med Educ. 1980;55(5):453-5.

43. Steinman MA, Shlipak MG, McPhee SJ. Of principles and pens: attitudes and practices of medicine housestaff toward pharmaceutical industry promotions. Am J Med. 2001;110(7):551-7. DOI 10.1016/S00029343(01)00660-X.

44. Hodges B. Interactions with the pharmaceutical industry: experiences and attitudes of psychiatry residents, interns and clerks. CMAJ. 1995;153(5):553-9.

45. Dana J, Loewenstein G. A Social Science Perspective on Gifts to Physicians From Industry. JAMA. 2003;290(2):252-5. DOI 10.1001/jama.290.2.252.

46. Steinbrook R. Physician-industry relations, Will fewer gifts make a difference? N Engl J Med. 2009;360(6):5579. DOI 10.1056/NEJMp0900143.

47. Waud DR. Pharmaceutical promotions-a free lunch? $N$ Engl J Med. 1992;327(5):351-3. DOI 10.1056/ NEJM199207303270511.
48. DeAngelis CD. Conflicts of interest in medical practice and their costs to the nation's health and health care system. Milbank Q. 2014;92(2):195-8. DOI 10.1111/1468-0009.12052.

49. Sah S, Fugh-Berman A. Physicians under the Influence: Social Psychology and Industry Marketing Strategies. J Law Med Ethics. 2013;41(3):665-72. DOI 10.1111/jIme. 12076 .

50. Cialdini RB. Influence: The new psychology of modem persuasion. New York: Quill; 1984.

51. Sah S. Conflicts of Interest and Your Physician: Psychological Processes That Cause Unexpected Changes in Behavior. J Law Med Ethics. 2012;40(3):482-7. DOI 10.1111/j.1748-720X.2012.00680.x.

52. Avorn J, Chen M, Hartley R. Scientific versus commercial sources of influence on the prescribing behavior of physicians. Am J Med. 1982;73(1):4-8.

53. Brennan TA, Rothman DJ, Blank L, Blumenthal D, Chimonas SC, Cohen JJ, et al. Health Industry practices That Create Conflicts of Interest. JAMA. 2006;295(4):429-33. DOI 10.1001/jama.295.4.429.

54. Marcia A. The truth about the drug companies: how they deceive us and what to do about it. New York: Random House; 2004.

55. Pronin E, Lin DY, Ross L. The Bias Blind Spot: Perceptions of Bias in Self Versus Others. Psychol Rev. 2004;111(3):781-99. DOI 10.1037/0033295X.111.3.781.

56. Festinger L. A theory of cognitive dissonance. Stanford: university press; 1962.

57. Chimonas S, Brennan TA, Rothman DJ. Physicians and Drug Representatives: Exploring the Dynamics of the Relationship. J Gen Intern Med. 2007;22(2):184-90. DOI 10.1007/s11606-006-0041-z.

58. Department of Health and Human Services, Office of Inspector General. Prescriptión drug advertisements in medical Journals [inernet]. [Accessed on 2019 March 8th]. Available at: https://bit.ly/2VO9DDj

59. Department of Health and Human Services. Office of Inspector General. Prescription Drug Promotion Involving Payments and Gifts.[internet]. [Accessed on March 8th, 2019] available at: https://bit. $\mathrm{Iy} / 2 \mathrm{u} 18 \mathrm{cFB}$

60. PhRMA [internet]. Washington: PhRMA; c2008. [Accessed on March $\left.8^{\text {th }}, 2019\right]$. Available at: https://onphr. $\mathrm{ma} / 2 z w n 15 T$ 
61. Agrawal S, Brennan N, Budetti P. The Sunshine Act Effects on Physicians. N Engl J Med. 2013;368(22):20547. DOI 10.1056/NEJMp1303523.

62. Lenzer J. Two years of sunshine: has openness about payments reduced industry influence in healthcare? BMJ. 2016,354:i4608. DOI 10.1136/bmj.i4608.

63. Benkimoun P. French "sunshine act" sheds little light on drug industry's payments to doctors, say critics. BMJ. 2013;346:f3762-f. DOI 10.1136/bmj.f3762.

64. Adlington K, Godlee F. Disclosure UK: transparency should no longer be an optional extra. BMJ. 2016;354:i3730. DOI 10.1136/bmj.i3730.

65. Consejo General de Colegios Médicos de España. Ética de la relación profesional del médico con la industria farmacéutica y las empresas sanitarias [Internet]. [accessed on March 8th, 2019] . Available from: https://bit.1y/2u5voTrf

66. Rojo-Abril M, Fragoso de Castro J, Balmy S, CaboDominguez L, Diaz-Trujillo L, Escudero-Galan N, et al. Primera Guía de Buenas Practicas de Información Médica en la Industria Farmacéutica: experiencia de trabajo. España: APDIS; 2016.
67. Lifshitz A, Halabe J, Jasso L, Frati A, Alva C, Arrieta Ó, Burgos R, et al. Comité de Ética y Transparencia en la Relación Médico-Industria (CETREMI); Recomendaciones para los médicos en su relación con la industria farmacéutica. Gac Med Mex. 2016;152:295.

68 Colombia. Ministerio de Salud y Protección Social. Resolución 2881 Transparencia en el sector farmacéutico y de tecnologías en salud [internet]. [Consultado mar 17 2019]. Disponible en: http://cort.as/-LQR5

69. Steinbrook R. Physicians, Industry Payments for Food and Beverages, and Drug Prescribing. JAMA. 2017;317(17):1753-54. DOI 10.1001/jama.2017.2477.

70. McCormick BB, Tomlinson G, Brill-Edwards P, Detsky AS. Effect of Restricting Contact Between Pharmaceutical Company Representatives and Internal Medicine Residents on Posttraining Attitudes and Behavior. JAMA. 2001;286(16):1994-9.

71. Gómez-Córdoba AI, Latorre-Santos C, Carreño JN. Dilemas éticos en las relaciones entre la industria farmacéutica y los profesionales de la salud. Pers Bioet. 2007;11(1). 